\title{
A new horizon for epigenetic medicine?
}

\author{
Cell Research (2013) 23:326-328. doi:10.1038/cr.2012.136; published online 18 September 2012
}

Histone lysine demethylases are chromatin modifiers that play important roles in many pathological processes such as inflammation and cancer, making them potentially attractive drug targets. In a recent study, Kruidenier et al. provided proof of concept by identifying chemical matters that inhibit demethylation mediated by the two related histone H3 lysine 27 demethylases, KDM6A and $6 \mathrm{~B}$ (UTX and JMJD3). The KDM6 inhibitor shows remarkable substrate selectivity and can inhibit transcription of a plethora of proinflammatory genes in cell culture by altering $\mathrm{H} 3 \mathrm{~K} 27 \mathrm{me} 3$ level at some of the KDM6 target genes.

In eukaryotic cells, DNA is packaged into chromatin whose basic units are nucleosomes. A nucleosome is consisted of $147 \mathrm{bp}$ nucleotides wrapped around a histone octamer, which is composed of two copies each of histone H2A, H2B, $\mathrm{H} 3$ and H4. Both DNA and histones are subjected to covalent chemical modifications, which impact chromatin organization and function. Recent mass spectrometry analysis identified more than a dozen different types of post-translational modifications on histone tails [1]. Among them, lysine methylation is one of the most extensively studied modifications, including histone H3 lysine 4 (H3K4), H3K9, H3K27, H3K36 and H4K20. Methylation at these lysine residues has been shown to play a role in transcription, DNA recombination and repair. Histone methylation is regulated by a plethora of methyltransferases and demethylases, which collectively regulate histone methylation dynamics. Histone dem- ethylases are composed of two classes, the flavin-dependent amine oxidases and iron- and $\alpha$-ketoglutarate-dependent dioxygenases, which share the highly related Jumonji (Jmj) C catalytic domain [2]. The latter class is composed of $\sim 20$ confirmed demethylases, which target different methylated lysine residues on histones [2]. Importantly, classic human genetic studies as well as more recent sequencing efforts of human disease genomes powered by next-generation DNA sequencing identified potential causal mutations in numerous chromatin modifying enzymes, including histone methyltransferases and demethylases [3-7]. These discoveries raise the exciting possibility that targeting chromatinmodifying enzymes may be a powerful means to combat human diseases.

H3K27 methylation is mediated by the Polycomb Repressive Complex 2 (PRC2), which is a multi-component enzymatic complex with EZH2 being the catalytic subunit. H3K27 tri- and dimethylation is reversed by a subfamily of two related JmjC domain-containing histone demethylases, UTX and JMJD3 (KDM6A and 6B) [2]. H3K27 di-methylation can also be demethylated by another related JmjC domain-containing demethylase KIAA1718 (KDM7A) [2]. H3K27 methylation has been shown to play important roles in development and differentiation. $\mathrm{H} 3 \mathrm{~K} 27 \mathrm{me} 3$ plays a critical role in the regulation of the Hox genes, which control animal anteriorposterior development. Consistently, loss of the H3K27me3 demethylase UTX results in a significant posterior development defect in zebrafish [8]. In embryonic stem cells, H3K27 and H3K 4 trimethylation co-exist and form what is so called "bivalent domains" on a subset of critical differentiationspecific genes. The bivalent domains are thought to poise genes for activation in response to appropriate developmental and differentiation cues. The resolution of the bivalent domains is likely to be mediated by the H3K4 and H3K27 histone demethylases, respectively.

When H3K27 trimethylation regulation goes awry, diseases ensue. For instance, overexpression of the H3K27 trimethyl methylase EZH2 is one of the hallmarks of prostate and breast cancer $[9,10]$. More recent studies also identified activating mutations of EZH2 in follicular lymphoma and diffuse large B cell lymphoma [7, 11]. Consistently, somatic mutations of the H3K27me3 demethylase UTX have been found in a number of caner types $[3,5,6]$. These findings indicate the importance of this mark as well as the corresponding methyltransferase EZH2 and the UTX/JMJD3 demethylases in tumorigenesis.

In addition to cancer, H3K27 methylation has also been featured prominently in inflammatory response. In fact, one of the first reports identifying JMJD3 as a histone $\mathrm{H} 3 \mathrm{~K} 27 \mathrm{me} 3$ demethylase documented a rapid JMJD3 induction by proinflammatory stimuli [12], and a follow-up study shows that JMJD3 is recruited to the transcription start sites (TSS) of the majority of lipopolysaccharide (LPS)-induced genes [13]. These studies suggest that modulating JMJD3 demethylase activity by small molecules may be one way to curtail inflammation. However, this possibility is complicated by a number of factors. First, the followup study suggests that regulation of 
the proinflammatory genes by JMJD3 may be independent of its demethylase activity [13]. Second, given the degree of sequence similarity among the JmjC domains of histone demethylases, it was unclear whether it is realistic to generate small molecules with sufficient substrate specificity. Now both of these issues have been answered by a recent elegant study by Kruidenier, Lee, Wilson and colleagues [14].

Kruidenier and colleagues combined high-throughput screens with structureguided design to identify chemical compounds that specifically inhibit the JMJD3 demethylase activity. They screened a $\sim 2$ million GSK compound library and obtained a number of weak hits. In parallel, they generated co-crystals of the JmjC domain plus the adjacent GATA-like zinc finger together with the H3K27 tri-methylated peptide. A $2.5 \AA$ co-crystal structure allowed them to define the contact sites on both the histone peptide and the catalytic domain. Leveraging the co-crystal structure information, the authors optimized the initial hits and were able to obtain a relatively potent lead compound GSK-J1, which has a half-maximum inhibitory concentration of $60 \mathrm{nM}$. Further efforts at delineating the co-crystal structure of the JMJD3 catalytic domain bound by GSK-J1 revealed the critical contact sites of GSKJ1 within the catalytic domain, which helped define the inhibitory mechanism of GSK-J1, i.e., it is competitive with the co-factor $\alpha$-ketoglutarate but not the substrate. The JMJD3 catalytic domain and GSK-J1 co-crystal structure also suggested strategies to modify GSK$\mathrm{J} 1$ for immobilization. The modified compound, GSK-J3, can be attached to sepharose beads without losing its activity. This results in a KDM6 probe, which was then used to capture endogenous JMJD3. In such an experiment, the author found that the immobilized GSK-J3 probe pulled down only JMJD3 from phorbol myristate acetate-stimulated HL-60 monocytic cells, again indicating specificity of this compound. However, GSK-J3 also can pull down the related enzyme UTX, suggesting that this compound does not distinguish JMJD3 from its related enzyme UTX. Importantly, GSK-J1 showed no activity towards a number of other demethylases such as the H3K9/K36 demethylases JMJD2A$\mathrm{E}$, as well as 60 chromatin regulators including histone deacetylases and 100 kinases. More will be learned about the specificity of GSK-J1 when additional $\mathrm{JmjC}$ demethylases are included in the specificity panel in the future, especially KIAA1718 (KDM7A), which demethylates H3K27me1/2 [2]. Regardless, the current data suggest impressive substrate selectivity of GSK-J1, especially considering the high degree of sequence homology among JmjC domains of different subfamilies of demethylases. Importantly, this finding shows that specific chemical inhibitors can be identified for specific subfamilies of demethylases, although it might prove difficult to develop chemical matters that can distinguish members of the same subfamily (such as JMJD3 and UTX).

As discussed earlier, JMJD3 has been shown to play a role in the inflammatory response. Intriguingly, a more recent study by De Santa et al. found that in the LPS-treated macrophages, although JMJD3 binds to the TSS of many targets, most of them have no detectable H3K27me3 [13]. On some genes, the H3K27me3 level did go down in response to LPS stimulation, but it was thought to be due to nucleosome depletion [13]. Interestingly, when Kruidenier and colleagues applied the cell permeable form of GSK-J1, i.e., GSK-J4, to LPS-stimulated human primary macrophages, they found that GSK-J4 inhibited 16 of 34 LPS-induced cytokines. They further demonstrated that the inhibitory effect on one of these cytokines, TNF- $\alpha$, can be mimicked only when both JMJD3 and UTX were inhibited by RNAi, indicating that both enzymes are involved in the TNF- $\alpha$ production. This finding supports the notion, which was developed based on the pull-down result, that GSK-J4 inhibits the activity of both JMJD3 and UTX. Furthermore, chromatin immunoprecipitation analysis shows that the inhibitor prevented the LPS-induced H3K $27 \mathrm{me} 3$ loss on the TNFA TSS. Together, these results demonstrate that the demethylase activity of JMJD3 and UTX is required for their functions in the inflammatory response. In the De Santa study [13], although TNFA was bound by JMJD3, it was not on the list of the H3K27me3-enriched genes before LPS stimulation, which could be due to H3K27me3 epitope masking. A more recent study supports the importance of the JMJD3 demethylase activity in regulating the expression of a subset of its direct target genes [15].

In summary, the development of KDM6-specific chemical inhibitors by Kruidenier and colleagues indicates that it is possible to generate subfamily member-specific demethylase inhibitors, thus paving the way for the hunt for inhibitors of other subfamily members, some of which have been demonstrated to play roles in other human diseases such as cancer. In addition, the availability of specific chemical inhibitors also empowers mechanistic investigation as nicely illustrated in this study where the inhibitors were used effectively to demonstrate the importance of JMJD3/ UTX-mediated demethylation in the inflammatory response.

\section{Shuzhen Chen ${ }^{1}$, Yang Shi ${ }^{1}$ \\ ${ }^{1}$ Division of Newborn Medicine and Program in Epigenetics, Department of Medicine, Bos- ton Children's Hospital and Department of Cell Biology, Harvard Medical School, Boston MA, 02115, USA \\ Correspondence: Yang Shi \\ E-mail: yshi@hms.harvard.edu}

\section{References}

1 Tan M, Luo H, Lee S, et al. Cell 2011; 146:1016-1028.

2 Greer EL, Shi Y. Nat Rev Genet 2012; 
13:343-357.

3 van Haaften G, Dalgliesh GL, Davies H, et al. Nat Genet 2009; 41:521-523.

4 Dalgliesh GL, Furge K, Greenman C, et al. Nature 2010; 463:360-363.

5 Gui Y, Guo G, Huang Y, et al. Nat Genet 2011; 43:875-878.

6 Grasso CS, Wu YM, Robinson DR, et al. Nature 2012; 487:239-243.
7 Morin RD, Johnson NA, Severson TM, et al. Nat Genet 2010; 42:181-185.

8 Lan F, Bayliss PE, Rinn JL, et al. Nature 2007; 449:689-694.

9 Varambally S, Dhanasekaran SM, Zhou M, et al. Nature 2002; 419:624-629.

10 Kleer CG, Cao Q, Varambally S, et al. Proc Natl Acad Sci USA 2003; 100:11606-11611.

11 Yap DB, Chu J, Berg T, et al. Blood 2011;
117:2451-2459.

12 De Santa F, Totaro MG, Prosperini E, et al. Cell 2007; 130:1083-1094.

13 De Santa F, Narang V, Yap ZH, et al. EMBO J 2009; 28:3341-3352.

14 Kruidenier L, Chung CW, Cheng Z, et al. Nature 2012; 488:404-408.

15 Chen S, Ma J, Wu F, et al. Genes Dev 2012; 26:1364-1375. 